\title{
ON THE COMPARATIVE PROTHROMBIC ACTIVITY OF HUMAN AND DOG BLOOD ${ }^{1}$
}

\author{
By ARMAND J. QUICK AND CLARA V. HUSSEY \\ (From the Department of Biochemistry, Marquette University School of Medicine, \\ Milwankee, Wis.)
}

(Submitted for publication April 14, 1951 ; accepted June 18, 1951)

Soon after the introduction of the one- and twostage methods for determining prothrombin, it was noted that while the results obtained by the two procedures generally agreed fairly well, marked discrepancies occurred in certain specific instances. Particularly puzzling has been the observation that the prothrombic activity of human blood as compared with dog blood, when measured by the two tests, is markedly different. Warner, Brinkhous and Smith (1) reported that the prothrombin concentration in dog blood is 350 units per cc. and in human blood 295 units. More recently Murphy and Seegers (2) found 190 to 205 units in dog and 290 to 315 in human blood. Mann and his associates $(3,4)$ reported that the average for dog blood is 183 units per cc. and for human blood 319 units. These findings indicate that the prothrombin concentration as measured by the two-stage method is approximately the same in the two species. When determined by the one-stage method, the prothrombic activity of dog blood, in marked contrast, is many-fold higher than in human blood (5). The reason for this divergency has not been satisfactorily explained, but obviously as long as this problem remains unsolved, a marked gap in the knowledge concerning prothrombin remains. In the present investigation human and dog bloods were studied by means of the prothrombin consumption test and by the adsorption and elution technique for determining prothrombin with the aim of obtaining new data that might be helpful in arriving at a better understanding of what constitutes prothrombic activity.

\section{METHODS}

The prothrombin consumption time. The directions as previously outlined were followed $(6,7)$. In carrying out the prothrombin consumption test on serum from

1 This work was supported by a grant from the Division of Research Grants and Fellowships of the National Institutes of Health, U. S. Public Health Service. platelet-poor plasma, blood is collected with a siliconecoated needle and syringe, and immediately transferred to silicone-coated test tubes immersed in an ice bath. After the tubes are chilled for 10 minutes, they are centrifuged in an Angle Centrifuge at 8000 r.p.m. for 10 minutes. One cc. of the plasma (having a platelet count less than 3000 per mm.) is transferred to a glass test tube and placed in a water bath at $37^{\circ} \mathrm{C}$. The fibrin is removed as it forms by wrapping it about a glass stirring rod. When the coagulation of fibrinogen is complete, the serum is tested every five minutes for its prothrombic activity. Usually it becomes constant in 15 minutes. The prothrombin time is determined as follows: To a mixture consisting of $0.1 \mathrm{cc}$. deprothrombinized oxalated rabbit plasma, 0.1 cc. thromboplastin and 0.1 cc. $0.02 \mathrm{M} \mathrm{CaCl}$ solution, $0.1 \mathrm{cc}$. of serum is added by blowing from a pipette. The formation of the clot is accurately timed.

The determination of prothrombin by the adsorption and elution technique as developed by Stefanini and the writer (8) was followed. In this test the prothrombin is adsorbed with tricalcium phosphate and then eluted with sodium citrate. Since one volume of eluate contains the prothrombin of 10 volumes of plasma, the eluate is mixed with nine volumes of deprothrombinized rabbit plasma for assaying its prothrombic activity. Rabbit plasma has been found to be a satisfactory assay medium because it has a high and constant concentration of labile factor.

\section{RESULTS}

A comparative study of the prothrombic activity of human and dog plasmas and sera are recorded in Table I. The prothrombin time of normal human plasma is 12 seconds and of dog plasma six seconds. The same values are obtained when determined by the adsorption and elution procedure. On storing oxalated human and dog plasma in an open container at $4^{\circ} \mathrm{C}$., the prothrombin time steadily increases but in human plasma the rise is much more rapid. If deprothrombinized rabbit plasma, which is a rich source of labile factor, is added to the stored human plasma, the prothrombin time is decreased to eight seconds. Likewise, the eluate obtained from aged human plasma yields a prothrombin time of eight seconds. When the labile factor is restored in 
TABLE I

The comparative prothrombic activity of dog and human plasma and serum

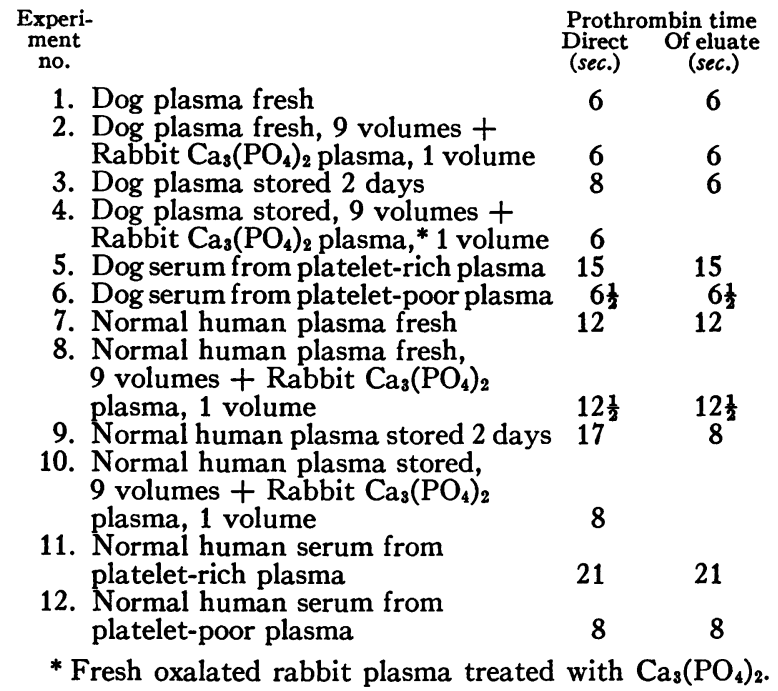

stored dog plasma, the prothrombin time again becomes six seconds; likewise the eluate has this value. There is, however, no increase in prothrombic activity during storage.

When platelet-rich human or dog plasma is clotted, the serum has a comparatively marked decrease in prothrombic activity. If the plasma is clotted after the removal of platelets, human serum has a prothrombin time of about eight seconds while dog serum has a value of six and one-half seconds. Native plasma, either platelet-rich or poor, from a severe hemophiliac, when clotted in glass yields a serum that has a prothrombin time of eight to eight and one-half seconds. In every instance the direct prothrombin time agrees with that obtained by the adsorption and elution procedure.

\section{DISCUSSION}

The simplest explanation for the difference of the prothrombin times of dog and human plasmas is that the former contains a much higher concentration of prothrombin than does human plasma. This conclusion is supported by the finding that on assaying the eluates of dog and human plasmas, it is found that their prothrombin times are six and 12 seconds respectively-the same as found by the direct one-stage test.

Although it has been shown that increasing the labile factor above the minimum level required for optimum prothrombin time does not further shorten it (9), it may still be claimed that the higher concentration of labile factor in dog blood might be responsible for the faster clotting time. When the prothrombin is determined by the adsorption and elution procedure, the labile factor is not a variable, because in the assay of the eluate, a standard amount of deprothrombinized rabbit plasma, which has a high concentration of labile factor, is employed. The close agreement between the results of the direct one-stage and the adsorption and elution method is good evidence that the labile factor does not influence the results of the one-stage method unless it is below the minimal requirement.

When normal dog blood clots, the prothrombin time of the serum increases because most of the prothrombin is converted to thrombin. In the experiment recorded in Table I, Exp. 5, the prothrombin time of the serum is 15 seconds. By means of the prothrombin curve for dog blood obtained by saline as a diluent (7), one can estimate that over 90 per cent of the prothrombin in this serum is consumed. In marked contrast, when platelet-poor plasma clots, the prothrombin time of the serum is practically the same as that of the plasma (Table I, Exp. 6). On account of the lack of platelets, so little thromboplastin apparently becomes available that only a very small amount of prothrombin is consumed. It is to be noted that in serum from both platelet-rich and poor plasma the prothrombin time by the direct one-stage method agrees with the values obtained by the adsorption and elution test. When oxalated dog plasma is stored, the prothrombin time slowly increases, but the eluate from this aged plasma had a prothrombin time of six to six and one-half seconds (Table I, Exp. 3) which clearly shows that no significant change in prothrombin occurs in dog plasma during short periods of storage.

When normal human blood clots, the serum has a prothrombin time which usually ranges from 15 to 30 seconds. This illustrates that, as in dog blood, much of the prothrombin is consumed during clotting. Fresh native plasma depleted of platelets by high centrifugation when clotted in glass yields a serum which has a prothrombin time of about eight seconds (Table I, Exp. 12). Significantly, the serum obtained from the clotting of 
TABLE II

The changes in prothrombic activity observed in hemophilic blood on storage and after clotting

$\begin{array}{lll}\begin{array}{c}\text { Experi- } \\ \text { ment } \\ \text { no. }\end{array} & \begin{array}{c}\text { Prothrombin time } \\ \text { Direct } \\ \text { (sec.) }\end{array} & \begin{array}{c}\text { Of eluate } \\ \text { (sec.) }\end{array} \\ \text { 1. Hemophilic plasma fresh* } & 12 & 12 \\ \text { 2. Hemophilic plasma stored 2 days } & 18 & 8 \\ \text { 3. Hemophilic plasma stored, } & & \\ \begin{array}{l}\text { 9 volumes + Rabbit Cas }\left(\mathrm{PO}_{4}\right)_{2}, \\ \text { 1 volume }\end{array} & 8 & \\ \text { 4. Hemophilic serum from platelet- } & 8-9 & 8-9 \\ \begin{array}{l}\text { rich plasma } \\ \text { 5. Hemophilic serum from platelet- } \\ \text { poor plasma }\end{array} & 8-9 & 8 \\ \text { * From a severe hemophiliac. } & 8 & \end{array}$

plasma of a severe hemophilic subject likewise has a prothrombin time of eight seconds even when the platelets are not diminished (Table II, Exps. 4 and 5). Furthermore, both normal and hemophilic blood when stored 24 to 48 hours have a prothrombin time of approximately eight seconds. Thus, a marked and relatively fixed increase in prothrombic activity occurs in sera from platelet-free normal plasma, from hemophilic blood or plasmas and in stored normal and hemophilic blood. In none of these sera or plasmas is more than a very small quantity of prothrombin consumed; therefore, nearly all of the prothrombin remains. This, however, does not account for the striking increase in prothrombic activity. The most direct and simplest explanation is that an actual increase in prothrombin occurs. For this to happen one may postulate that plasma contains an inactive or precursor form of prothrombin. Bordet (10) developed the hypothesis that all prothrombin (serozyme) in circulating blood is in an inactive form. Quick and Stefanini (9) concluded that in human plasma part of the prothrombin is active, but a much greater proportion is in the precursor state. This inactive prothrombin, like active prothrombin, is completely adsorbed and eluted. The eluate in contact with glass likewise increases markedly in prothrombic activity, presumably because the precursor is gradually changed to active prothrombin. Recently it was found that the quantity of thromboplastin required for maximum prothrombic activity varies with the prothrombin concentration (11). When the prothrombin is reduced by means of Dicumarol or vitamin $\mathrm{K}$ deficiency, the thromboplastin requirement decreases. Considerably more thromboplastin is needed to obtain the minimum prothrombin time of stored plasma than of fresh plasma, which suggests that an increase of prothrombin itself occurs.

If the increase in prothrombic activity is not due to an increase of active prothrombin, then it becomes necessary to postulate that an accelerator is generated to account for the decrease of the prothrombin time from the normal of 12 seconds to eight seconds. Alexander and his associates (12) have attempted to establish that such a specific agent exists. They have named it spca (serum prothrombin converting accelerator) because they maintain that the prothrombic activity of serum is due largely to the evolution of this factor during clotting. If their contentions are correct, then the marked increase of prothrombic activity found in the serum from hemophilic or from thrombocytopenic blood should be due to spca, but according to their findings this factor is low in these sera $(13,14)$. From this it would follow that the marked hyperprothrombic activity in these sera is not due to the evolution of an accelerator but to an actual increase in prothrombin.

According to Alexander, spca, like prothrombin, is adsorbed by $\mathrm{BaSO}_{4}$ and eluted with sodium citrate. In isolating spca, they allow blood to clot and treat the serum with $\mathrm{BaSO}_{4}(20 \mathrm{mgm}$. per $1 \mathrm{cc}$. serum). From their statement (15): "most of the prothrombic activity was in the supernatant" after adsorption with $\mathrm{BaSO}_{4}$, one must infer that these investigators assume that differential adsorption occurs in which spca is separated from prothrombin. On repeating their procedure, it has been found that as much as two-thirds of the prothrombic activity of serum is removed by $20 \mathrm{mgm}$. of $\mathrm{BaSO}_{4}$ per $1 \mathrm{cc}$. (16). There is therefore a strong probability that the separation of spca and prothrombin is more apparent than real and that the product considered to be the accelerator contains an undetermined amount of prothrombin. Alexander and Landwehr's (17) finding that the evolution of spca during storage is small in various hypoprothrombic states might well be explained by a low concentration of prothrombin in the accelerator preparation. To determine how much of the activity is due to prothrombin and how much to an accelerator becomes not only a formidable but a speculative problem.

Thus, while the possibility of an accelerator factor should not be ignored, it seems simpler and 
perhaps more firmly supported by experimental evidence that the hyperprothrombic activity in the conditions discussed is due to an actual increase in active prothrombin. The statement of Stefanini and Crosby (18) that the prothrombin time of serum is not influenced by the activation of the precursor of prothrombin in less than two hours after the completion of clotting is not in accord with the findings of the writers. In the clotting of native human plasma the prothrombin time of the serum attains a constant value in about 15 minutes after all the fibrinogen has been converted to fibrin. In oxalated plasma, the conversion of inactive prothrombin to the active state is slow. At $4^{\circ} \mathrm{C}$. the reaction usually requires more than 24 hours to go to completion, even in contact with a glass surface, and in a silicone-coated container it even takes considerably longer. This permits the removal of both prothrombin and prothrombinogen from oxalated plasma by adsorption with tricalcium phosphate, and the subsequent recovery of both forms by elution with sodium citrate.

The finding that neither storage nor the clotting of platelet-poor dog plasma causes an increase of prothrombic activity such as is observed in human plasma, makes it unlikely that dog blood contains a prothrombin converting factor of the type of spca. One is at a loss, therefore, to find a better explanation for the high prothrombic activity of dog blood than that it contains a much higher concentration of active prothrombin than does human blood, but has little or no inactive prothrombin.

\section{SUMMARY}

The comparative prothrombic activity of dog and human blood were studied by means of the one-stage prothrombin test, the prothrombin consumption test and the adsorption and elution technique for determining prothrombin.

It was found that the prothrombic activity of dog blood is much greater than that of human blood. When oxalated dog plasma is stored, the prothrombin time is increased due to loss of labile factor. When the latter is restored, the prothrombin time returns to the value obtained for fresh plasma. The serum from platelet-poor normal plasma has a prothrombin time only slightly less than that of plasma.
Human plasma on storage likewise shows a progressive decrease of prothrombic activity but when the labile factor is restored, the prothrombin time becomes shorter than that of the fresh plasma. A similar increase in prothrombic activity is found when normal platelet-poor plasma, or hemophilic plasma clots.

Possible explanations for these findings are discussed. It is concluded that dog blood contains a high concentrate of free or active prothrombin but little or no inactive prothrombin, whereas human plasma has a much smaller amount of free prothrombin, but a high concentration of inactive prothrombin (prothrombinogen), which in contact with a glass surface becomes converted to the active state.

\section{REFERENCES}

1. Warner, E. D., Brinkhous, K. M., and Smith, H. P., The prothrombin conversion rate in various species. Proc. Soc. Exper. Biol. \& Med., 1939, 40, 197.

2. Murphy, R. C., and Seegers, W. H., Concentration of prothrombin and Ac-globulin in various species. Am. J. Physiol., 1948, 154, 134.

3. Mann, F. D., Shonyo, E. S., and Mann, F. C., Effect of removal of the liver on blood coagulation. Am. J. Physiol., 1951, 164, 111.

4. Mann, F. D., Butt, H. R., and Hurn, M., Prothrombin in liver disease: a clinical evaluation of the two-stage method. Gastroenterology, 1948, 11, 221.

5. Quick, A. J., The prothrombin concentration in the blood of various species. Am. J. Physiol., 1941, $132,239$.

6. Quick, A. J., The coagulation mechanism with specific reference to the interpretation of prothrombin time and a consideration of the prothrombin consumption time. Am. J. Clin. Path., 1949, 19, 1016.

7. Quick, A. J., The Physiology and Pathology of Hemostasis. Lea \& Febiger, Philadelphia, 1951.

8. Quick, A. J., and Stefanini, M., The concentration of component $\mathrm{A}$ in blood, its assay and relation to the labile factor. J. Lab. \& Clin. Med., 1949, 34, 973.

9. Quick, A. J., and Stefanini, M., The state of component $A$ (prothrombin) in human blood; evidence that it is partly free and partly in an inactive or precursor form. J. Lab. \& Clin. Med., 1949, 34, 1203.

10. Bordet, J., The theories of blood coagulation. Bull. Johns Hopkins Hosp., 1921, 32, 213.

11. Quick, A. J., and Hussey, C. V., The influence of the concentration of thromboplastin on the prothrombin time of human and dog plasma. Proc. Soc. Exper. Biol. \& Med., 1951, 76, 732. 
12. Alexander, B., deVries, A., Goldstein, R., and Landwehr, G., A prothrombin conversion accelerator in serum. Science, 1949, 109, 545.

13. Alexander, B., and deVries, A., Studies on hemophilia. V. The coagulation defect in hemophilia with particular reference to the conversion of prothrombin to thrombin and the evolution of the prothrombin conversion accelerator. Blood, 1949, 4, 752.

14. Alexander, B., and deVries, A., A factor in serum which accelerates the conversion of prothrombin. to thrombin. III. Its relationship to the coagulation defect of thrombocytopenic blood. Blood, 1949, 4, 747.
15. Alexander, B., Goldstein, R., and Landwehr, G., The prothrombin conversion accelerator of serum (spca) : Its partial purification and its properties compared with serum Ac-globulin. J. Clin. Invest., 1950, 29, 881.

16. Unpublished findings.

17. Alexander, B., and Landwehr, G., Evolution of a prothrombin conversion accelerator in stored human plasma and prothrombin fractions. Am. J. Physiol., 1949, 159, 322.

18. Stefanini, M., and Crosby, W. H., Serum prothrombin time, a composite effect. An analysis of factors involved. Am. J. Clin. Path., 1950, 20, 1026. 